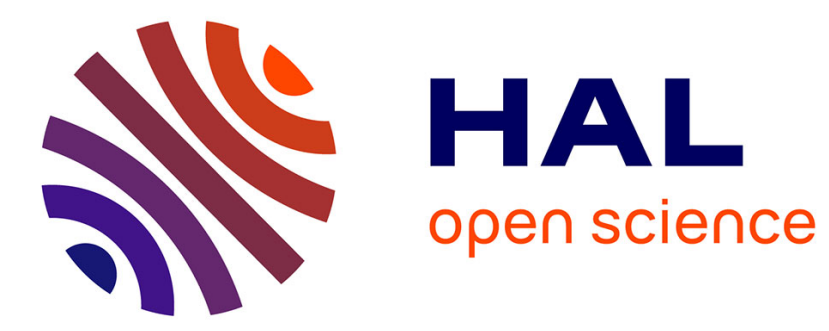

\title{
EXPERIMENTAL AND THEORETICAL STUDY OF ELASTIC WAVE DISPERSION IN A LIQUID BILAYER
}

J. Laperre, W. Thys

\section{- To cite this version:}

J. Laperre, W. Thys. EXPERIMENTAL AND THEORETICAL STUDY OF ELASTIC WAVE DISPERSION IN A LIQUID BILAYER. Journal de Physique IV Proceedings, 1992, 02 (C1), pp.C1-907C1-910. 10.1051/jp4:19921198 . jpa-00251163

\section{HAL Id: jpa-00251163 https://hal.science/jpa-00251163}

Submitted on 1 Jan 1992

HAL is a multi-disciplinary open access archive for the deposit and dissemination of scientific research documents, whether they are published or not. The documents may come from teaching and research institutions in France or abroad, or from public or private research centers.
L'archive ouverte pluridisciplinaire HAL, est destinée au dépôt et à la diffusion de documents scientifiques de niveau recherche, publiés ou non, émanant des établissements d'enseignement et de recherche français ou étrangers, des laboratoires publics ou privés. 


\title{
EXPERIMENTAL AND THEORETICALSTUDY OF ELASTIC WAVE DISPERSION IN A LIQUID BILAYER
}

\author{
J. LAPERRE and W. THYS \\ Katholieke Universiteit Leuven Campus Kortrijk, Interdisciplinary Research Center, B-8500 Kortrijk, \\ Belgium
}

\begin{abstract}
In this paper we analyse the dispersion of the normal propagation modes of a liquid bilayer in terms of the dispersion in the constituent layers with the proper boundary conditions. The analysis is done in the framework of an acoustic raymodel which leads to a transparent form of the dispersion relation. Although a liquid bilayer is of no direct practical use, the data analysis will prove to be of great help when studying solid multilayers.

Résumé Dans cet article nous analysons la dispersion des modes de Lamb d'une bicouche liquide, en partant des modes des monocouches isolées avec les conditions de bord adaptées. L'analyse est faite à l'aide d'un modèle à rayon, ce qui permet d'écrire la relation de dispersion dans une forme simple et transparente. Les conclusions et la façon d'analyser seront utiles pour comprendre la dispersion dans des multicouches solides.
\end{abstract}

\section{Introduction}

This paper is the first in a series on the dispersion of elastic waves in multilayers. Although a liquid bilayer is of no direct practical use, the fact that it is simple especially that there is no mode conversion, will allow us to understand as much as possible about the normal propagation modes. The data analysis developed for a liquid bilayer, will prove to be of great help when studying solid multilayers.

\section{Dispersion relation of a liquid bilayer}

The dispersion relation of a plate or a multilayer determines the normal propagation modes of the elastic system surrounded by air. More specifically, it determines the wavenumber of the free waves which can propagate unattenuated and without external excitation in the plate or the multilayer. The standard procedure [1] for obtaining the dispersion relation in a multilayer is rather mathematical and it does not provide much physical insight.

Inspired by the ray analysis in optics [2] [3] [4], we developed [5] an acoustic raymodel based on the interpretation of normal mode propagation in a multilayer as a wave guide phenomenon. The dispersion relation is obtained by requiring:

- that in order to fulfill the condition of translation invariance, the acoustic waves traveling in the guiding layer, are totally reflected on the boundaries;

- that the totally reflected waves form a self-sustaining traveling interference system.

Using these conditions, we construct and analyse in this paragraph, the dispersion relation of a liquid bilayer.

It consists of two plane liquid layers (labeled 1 and 2) on top of each other, and surrounded by air. The layers have a thickness $2 d_{1}$ and $2 d_{2}$, and a density $\rho_{1}$ and $\rho_{2}$ respectively (see figure 1 ). We assume that the longitudinal sound velocity $v_{1}$ is smaller in layer 1 than in layer 2 . Within the raymodel, the second 
requirement puts the following condition on the phase (see figure 1): the change in phase of a plane wave progressing from $A$ to $D$, is equal to the phase change along the ray path $A B C D$, within an integer multiple of $2 \pi$. This results in the following equation:

$$
2 d_{1} k_{1 z}+\frac{\pi}{2}+\Omega_{1,2}=n_{b} \pi
$$

$\frac{\pi}{2}$ and $\Omega_{1,2}$ are half of the change in phase of a plane wave propagating in layer 1 , when it is totally reflected on respectively air (index 0 ) and on layer $2 . \Omega_{1,2}$ can be calculated using the algorithm of Thomson [6]. $k_{1 z}$ is the $\mathrm{z}$-component of the wavevector in layer 1 ; it is given by:

$$
k_{1 z}=\frac{\omega}{v_{1}} \cos \theta_{1}
$$

where $\theta_{1}$ is angle of incidence of the acoustic ray on the boundaries and $\omega$ is the circular frequency of the plane wave.

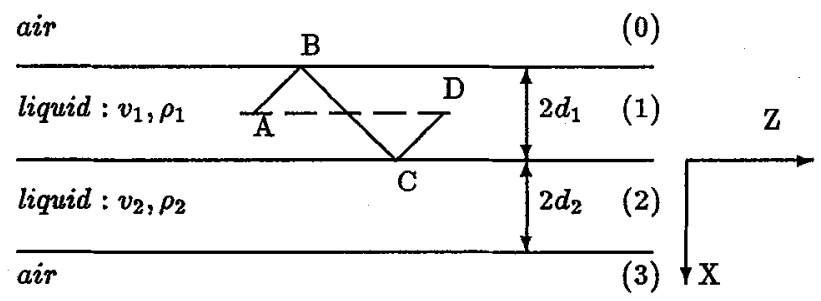

Figure 1: Identification of the liquid bilayer

It can be demonstrated that equation 1 is identical with the dispersion relation obtained by the standard procedure [5].

By solving equation 1 numerically, we obtain the angles $\theta_{1}$ for which at a given frequency, a normal mode can propagate unattenuated in the liquid layer. The phasevelocity $v_{L}$ of these modes is then given by Snell's law:

$$
v_{L}=\frac{v_{1}}{\sin \left(\theta_{1}\right)}
$$

In figure 2 we plotted as solid lines the real roots $\sin \left(\theta_{1}\right)$ of equation 1 as a function of frequency for the liquid bilayer with the following parameters: $v_{1}=2000 \mathrm{~m} / \mathrm{s}, v_{2}=5500 \mathrm{~m} / \mathrm{s}, 2 d_{1}=0.001 \mathrm{~m}, 2 d_{2}=0.003$ $\mathrm{m}$ and $\rho_{1}=\rho_{2}=1000 \mathrm{~kg} / \mathrm{m}^{3}$. Each curve on figure 2 corresponds to a particular value of the integer $n_{b}$ as indicated. We added BL to avoid confusion later on. These curves can be understood by studying wave dispersion in each layer separately, with the proper boundary conditions. As far as layer 1 is concerned, it is reasonable to assume that it is rigidly held on its boundary with layer 2 because the acoustic impedance (defined as $\rho v$ ) of layer 1 is smaller than that of layer 2. The other boundary of layer 1 may be considered as free. This results in the following dispersion relation for the isolated layer 1 with one boundary free and the other rigidly held:

$$
2 k_{1} d_{1}+\frac{\pi}{2}=n_{1} \pi
$$

Because the boundary conditions are different on both sides, the normal modes of layer 1 can not be catalogued as symmetric or antisymmetric. We therefore use the value of $n_{1}$ to number the modes.

Layer 2 may be considered as free on both sides; its dispersion relation is:

$$
2 k_{2} d_{2}=n_{2} \pi
$$

The modes of layer 2 are symmetric or antisymmetric, so that the notations $S$ or $A$ with $n_{2}$ as an index, can be used. In figure 2 we plotted equation 4 and equation 5 on top of the dispersion curves of the bilayer. Several interesting features appear immediately: 
- Above the critical angle $\theta_{c}\left(\sin \theta_{c}=0.36\right)$ of the interface between layer 1 and layer 2 , the dispersion curves of the bilayer follow more or less the dispersion curves of layer 1 being the layer with the smallest impedance. The zigzagging ray in layer 1 now generates a wave in layer 2 , which is exponentially decaying in the $\mathrm{z}$-direction.

- Near normal incidence and at low frequencies, the normal modes of the bilayer coincide with a mode of one of the isolated layers.

- At intermediate angles, mode coupling makes a mode of the bilayer switch to the next higher (BL3 for instance) or the next lower (BL4 for instance) mode of the layer which was resonant near normal incidence.

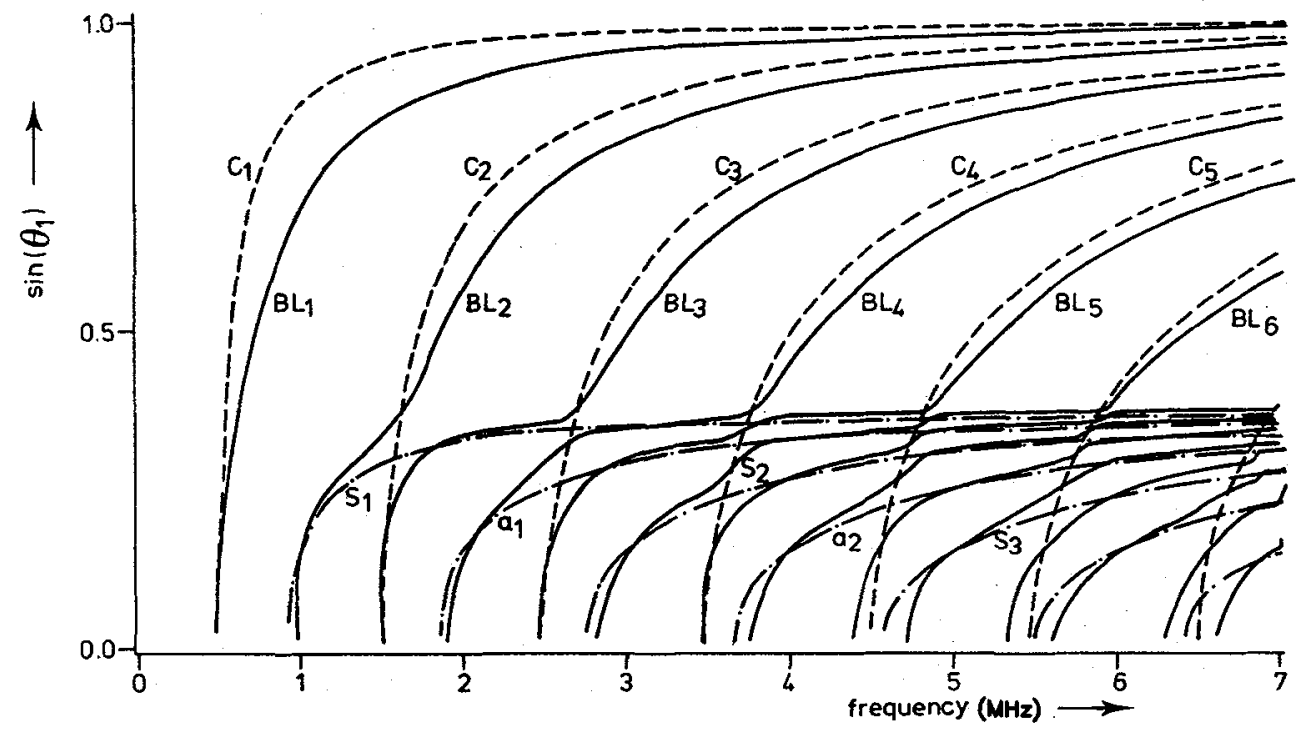

Figure 2: Dispersion curves of the liquid bilayer (solid lines) and of the constituent monolayers(dash lines and dot-dash lines)

These points and especially point three, can be made clear by numerically studying the longitudinal displacement $u_{z}$ in the bilayer and the phasejump $2 \Omega_{1,2}$ [7]. We concentrate on mode BL3. Near normal incidence layer 1 is resonant in its second mode. $2 \Omega_{1,2}$ is then zero so that the dispersion relation of the bilayer (equation 1) indeed corresponds to mode 2 of layer 1 . As we increase the frequency and pass through the resonance $S 1$ of layer 2 , half a wavelength is added to the oscillation layer 1 , so that the waveform now corresponds to the next higher mode of layer 1 . At the same time $2 \Omega_{1,2}$ changes to $-2 \pi$, so that the dispersion relation of the bilayer is then exactly the dispersion relation of mode 3 of layer 1.

In general we can conclude that, if near normal incidence the bilayer is tuned to a mode of one of the layers. Crossing a resonance of the other layer changes $\Omega$ by $\pi$ and switches the bilayer to the next higher or the next lower mode of the layer it was initially tuned to.

\section{An experiment}

One serious drawback of the raymodel used in the preceding paragraph, is that it does not account for mode conversion between longitudinal and transversal waves in the guiding layer. A verification of the conclusions of the preceding paragraph thus requires an experiment where mode conversion is negligable or nonexisting. A solid plate loaded with a thin liquid layer at normal incidence is a possible target. This can be realised by positioning a $0.61 \mathrm{~mm}$ thick aluminum plate close to a solid halfspace in a watertank. The distance between the plate and the halfspace determines the thickness of the liquid layer. The bilayer thus formed behaves, at normal incidence, very much like a liquid bilayer even though one of its constituents is a solid. The 
thickness of the water layer is varied between $100 \mu \mathrm{m}$ and $800 \mu \mathrm{m}$ by moving the reflector. It is measured with a micrometer attached to the frame.

With this setup we did the following experiment. We insonify the bilayer at normal incidence with a broadband ultrasonic transducer of $5 \mathrm{MHz}$, operating in pulse-echo mode.

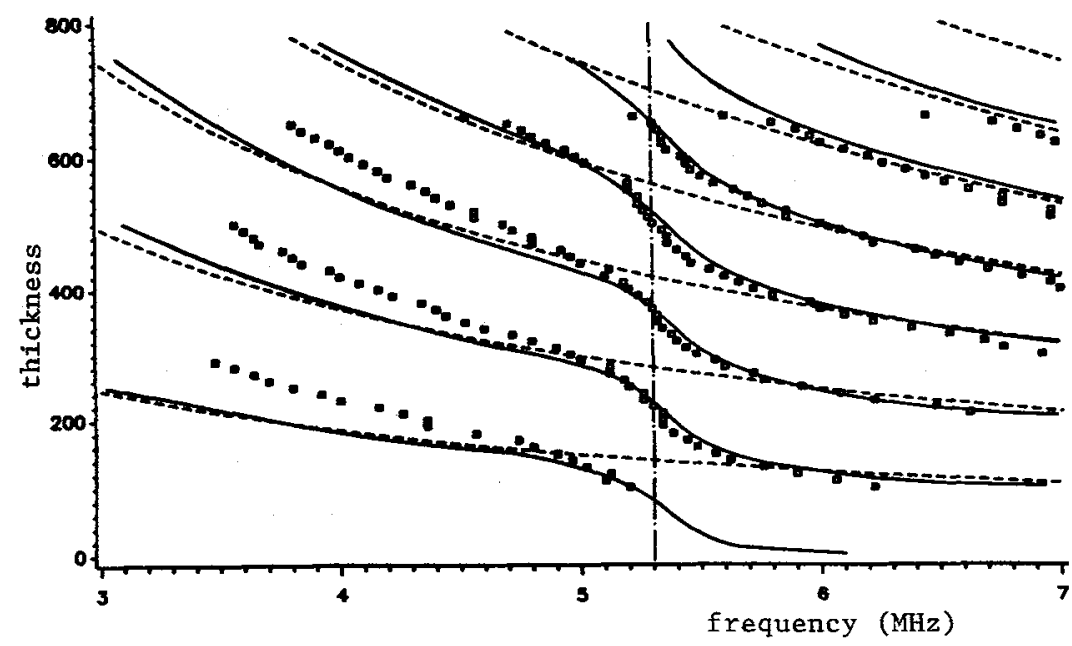

Figure 3: Dispersion data for the aluminum plate loaded with a thin water layer at normal incidence. The experimental points are plotted as $\square$ 's. The solid lines are the dispersion curves of the bilayer. The dashed lines are the dispersion curves of the water layer and the dashdot line corresponds to the resonance of the aluminum plate at $5.29 \mathrm{MHz}$

For each thickness of the water layer, the frequency analysis of the reflected pulse is recorded and the frequency of the reflection minima is determined. The angle of incidence being fixed and equal to zero degrees, we plot the experimental data ( $\square$ 's) in figure 3 as thickness of the water layer versus frequency. On the same figure we also plotted the theoretical dispersion curves. The solid lines represent the modes of the bilayer. The modes of the water layer rigidly held on both sides, are plotted as dash lines. The 0.61 $\mathrm{mm}$ thick aluminum plate behaves in the bilayer as a free plate, and has one resonance at $5.29 \mathrm{MHz}$ the frequency of which does not depend on the thickness of the liquid layer. It is represented on the figure 3 as a vertical line.

Inspection of this figure, clearly shows that coupling between the modes of the waterlayer and of the AL-plate, switches the dispersion curves of the bilayer to the next lower mode of the liquid layer. As in the preceding paragraph this can be explained by looking at the behaviour of $2 \Omega_{2,1}$ as a function of frequency.

The discrepancies between the experimental points and the numerical dispersion curves at low frequencies are not well understood.

\section{References}

[1] Brekhovskikh, L., Waves in layered media, Academic Press (1960).

[2] Kogelnik, H. and Weber,H.P., J. Opt. Soc. Am. 7,174(1974).

[3] Li, Y.F. and Lit, J.W.Y., J. Opt. Soc. Am. (A) 7,617 (1990).

[4] Snyder, A.W. and Love, J.D., Chapman and Hall, London 1983.

[5] Laperre, J. and Thys, W., Acustica 67,86 (1988).

[6] Thompson, W.T., J. Appl. Phys. 21, 89 (1950).

[7] Laperre, J. and Thys, W., To be published. 